\title{
The Epidemiology of Depression and the Evolution of Treatment
}

\author{
Robert M. A. Hirschfeld, MD
}

\begin{abstract}
Depression is a prevalent and pernicious disorder. About 1 in 5 US adults have at least 1 lifetime episode of major depression. Of those with depression, the majority will relapse over the long-term and many will have poor mental health outcomes and psychosocial disabilities. Over the past century, a range of treatments, including medications with varying mechanisms of action, have been developed to manage depression. Treatments from seizure therapies to an array of medications-amphetamine, tricyclic antidepressants, monoamine oxidase inhibitors, mixed-action antidepressants, selective serotonin reuptake inhibitors, and dual reuptake inhibitors - have evolved.

(J Clin Psychiatry 2012;73[suppl 1]:5-9)
\end{abstract}

D epression is a prevalent, pernicious, and undertreated disorder, despite the wide range of treatments currently available. By examining the evolution, use, and underuse of these treatments, clinicians may be better equipped to manage this condition.

\section{EPIDEMIOLOGY OF DEPRESSION}

Depression continues to be a highly prevalent disorder. In the United States, the prevalence of Diagnostic and Statistical Manual of Mental Disorders, Fourth Edition $(D S M-I V)^{1}$ depression was assessed in the National Comorbidity Survey Replication (NCS-R) study. ${ }^{2}$ In US adults, the 12-month prevalence rate of a major depressive episode was $8.3 \%$, and the lifetime prevalence rate was $19.2 \% .^{2}$ Depression usually starts in early adulthood, and the mean age at onset is 26 years. $^{2}$ The distribution of lifetime depression is fairly evenly spread across the age groups in early and middle age but decreases in those over 65 years of age (Figure 1). ${ }^{2}$ Depression occurs considerably more frequently among women than among men across all age groups.

\section{Clinical Course}

Several studies have examined the long-term course of depression. ${ }^{3,4}$ In the National Institute of Mental Health Collaborative Depression Study (CDS), ${ }^{3} 431$ patients from 5 US medical centers who met Research Diagnostic Criteria for major depressive disorder (MDD) were observed prospectively for 5 years. Using the Kaplan-Meier method, the researchers calculated cumulative probabilities of recovery at various intervals. According to the estimates, $54 \%$ of patients

\footnotetext{
From the Department of Psychiatry and Behavioral Sciences, University of Texas Medical Branch, Galveston.

This article is derived from the planning teleconference series "A Fresh Look at Monoamine Oxidase Inhibitors for Depression," which was held December 2011 through February 2012 and supported by an educational grant from Mylan Specialty L.P. (formerly known as Dey Pharma, L.P.).

Dr Hirschfeld has received royalties from Jones and Bartlett, has received honoraria from Merck Manual Editorial Board, and has received CME support from CME Outfitters and Nevada Psychiatric Association.

Corresponding author: Robert M. A. Hirschfeld, MD, Department of Psychiatry, 301 University Blvd, Galveston, TX 77555 (rohirsch@utmb.edu). doi:10.4088/JCP.11096su1c.01

(.) Copyright 2012 Physicians Postgraduate Press, Inc.
}

were likely to recover from an episode of MDD within 6 months, $70 \%$ of patients were likely to recover within a year, and $81 \%$ of patients were likely to recover by 2 years. ${ }^{3}$ This left 1 in 5 patients continuously depressed for 2 years, the majority of whom remained depressed for at least 5 years. These individuals never had 8 or more consecutive weeks without symptoms or with only 1 or 2 mild symptoms.

A prospective follow-up ${ }^{4}$ extended the CDS to 15 years or longer. ${ }^{3}$ Among the 380 participants who recovered from the index major depressive episode at some time during the 15 -year follow-up, $85 \%$ relapsed. Even among the individuals who had remained well for at least 5 years $(n=105)$ following recovery, $58 \%$ relapsed over the next 10 years. ${ }^{4}$ Among those not lost to follow-up, only 1 in 9 people had a single depressive episode, recovered, and then stayed well over the 15 years. ${ }^{4}$ Most patients who experienced an episode of depression were likely to have additional episodes throughout their lives.

\section{Psychosocial Toll}

The burden of depression is heavy. In a large community study in Upper Bavaria $(\mathrm{N}=838),{ }^{5}$ those individuals who had depressive symptomatology at baseline (18.1\%) had poor mental health outcomes, substantial psychosocial disability, and functional impairment throughout 25 years of follow-up.

Another analysis ${ }^{6}$ of the CDS data showed that patients with MDD had at least some symptoms more than half of the time (ie, 59\% of weeks) during 2 to 12 years of follow-up.

The World Health Organization has ranked MDD as the third leading cause of disease burden in the world and the leading cause of burden of disease in high- and middleincome countries. ${ }^{7}$ Depression accounts for $4.3 \%$ of total disability-adjusted life-years (DALYs) in the world.

\section{Unipolar Versus Bipolar Depression}

When evaluating a patient who presents with depression, assessing whether the individual has unipolar or bipolar depression is crucial. The clinical course of and treatment for MDD and bipolar disorder differ. Bipolar disorder is often misdiagnosed as MDD. The presence of bipolar disorder is easy to miss in patients with depression because many depressed patients with bipolar disorder fail to report prior manic or hypomanic episodes. 
- Depression is highly prevalent and is a leading cause of disability worldwide.

- Bipolar disorder is often misdiagnosed as major depression due to patients' underreporting of manic/hypomanic symptoms.

- Several medications are effective in treating major depression, including TCAs, MAOIs, SSRIs, and SNRIs.

In a recent study ${ }^{8}$ of the CDS cohort, 550 patients who were diagnosed with major depression (but not bipolar disorder) and had been followed for at least 1 year were screened for subthreshold manic symptoms. At intake, $22 \%$ endorsed 1 to 5 manic symptoms at levels below the threshold for a bipolar disorder diagnosis. Participants were then monitored for up to 31 years (mean duration $=17.5$ years). During this follow-up, about $20 \%$ of the sample experienced manic or hypomanic symptoms and were subsequently diagnosed with bipolar disorder. Hypomania occurred prospectively in $12.2 \%$ of the sample, and mania occurred prospectively in $7.5 \%$ of the sample. The symptoms most predictive of the switch to mania or hypomania were decreased need for sleep, increased energy, and increased goal-directed activity. Individuals who might, in the past, have been diagnosed with anxious depression or agitated depression should be assessed for increased energy that might be indicative of bipolar disorder.

\section{CATEGORIZATION OF DEPRESSIVE DISORDERS}

Reviewing the epidemiology of depression and the evolution of depression treatments is appropriate considering the categories of depression as defined by the American Psychiatric Association are undergoing changes in the forthcoming DSM-5. ${ }^{9}$

Proposed changes to the DSM categories of depression are shown in Table 1; these changes are still under discussion. It is suggested that within the category of depressive disorders, MDD as a single episode or recurrent disorder will be retained, but a new category, chronic depressive disorder, will be created and will include dysthymia. Premenstrual dysphoric disorder and mixed anxiety/depression, which appeared in Appendix B of the DSM-IV, will become subcategories of depressive disorders in the main body of the $D S M-5$ text. Changes to specifiers have also been proposed, including omitting the specifier "with chronic features" and adding the specifiers "with mixed features," "with anxiety," and "with suicide risk severity."

\section{EVOLUTION OF DEPRESSION TREATMENTS}

Over the last 100 years, treatments for MDD, particularly pharmacologic treatments, have advanced considerably (Figure 2).
Figure 1. Lifetime Prevalence of DSM-IV Depression in

US Adults by Gender and Age Group ${ }^{a}$

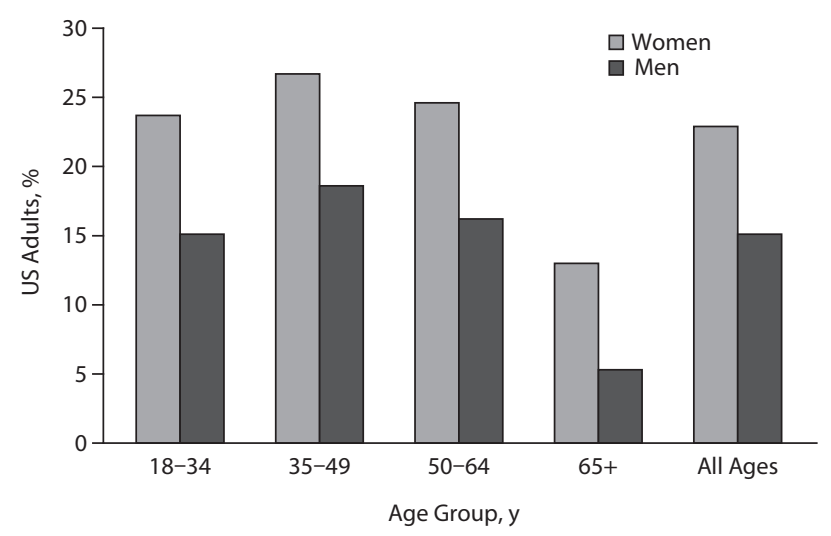

${ }^{\text {a}}$ Data from Kessler et al. ${ }^{2}$

Abbreviation: DSM-IV= Diagnostic and Statistical Manual of Mental Disorders, Fourth Edition.

\section{Seizure or Convulsive Treatments}

In the early 1930s, the first somatic treatments for depression emerged from serendipitous observations of patients undergoing treatment for other problems. ${ }^{10}$ While in Berlin treating patients with insulin for morphine withdrawal, Manfred Sakel noticed that insulin-induced hypoglycemic comas had the effect of calming patients with psychotic symptoms. He subsequently had success with insulininduced comas in patients with schizophrenia. Some patients experienced seizures during these comas.

Around the same time in Budapest, Ladislaus von Meduna induced seizures in psychotic patients with camphor, which had a positive effect on their psychiatric symptoms. He subsequently found that pentylenetetrazol (marketed as Metrazol and Cardiazol), a cardiac stimulant, was more reliable in inducing seizures than camphor. Among 26 patients with schizophrenia treated with camphor or Metrazol by Meduna, 10 recovered and 3 showed improvement. ${ }^{10}$

By 1937, both insulin coma and convulsive therapy were widely used. ${ }^{10}$ Some practitioners tried Metrazol in conjunction with insulin or on an alternating schedule; Metrazol monotherapy was safer and simpler than performing hypoglycemic shock therapy. While insulin-induced coma therapy required daily treatments followed by 5 hours of medical attention and carried a risk of prolonged coma, intravenous Metrazol could be quickly administered to patients 3 times per week.

In 1937 in Rome, Ugo Cerletti and Lucio Bini presented their work on electroconvulsive therapy (ECT) at a conference detailing how they induced seizures via electrical currents rather than with medication. ${ }^{10}$ While ECT was first used for treating psychosis, it was found to be more effective as a treatment for patients with affective symptoms. ${ }^{11}$ This resulted in the use of Metrazol and insulin-induced coma treatment declining and the use of ECT increasing. Electroconvulsive therapy was more convenient to administer, produced less severe convulsions, was less painful, and was less feared by patients than previous convulsive treatments. 


\begin{tabular}{|c|c|c|c|}
\hline \multicolumn{2}{|r|}{$D S M-I V$} & \multicolumn{2}{|r|}{ DSM-5 (Proposed) } \\
\hline \multirow[t]{2}{*}{ Code $^{\mathrm{b}}$} & Category & Code & Category \\
\hline & Mood disorders & & Depressive disorders \\
\hline \multirow{8}{*}{$\begin{array}{r}296 . x x \\
.2 x\end{array}$} & Depressive disorders & D 00 & Disruptive mood dysregulation disorder \\
\hline & Major depressive disorder & $\mathrm{D} 01$ & Major depressive disorder, single episode \\
\hline & Single episode & & Mixed \\
\hline & Chronic & & Catatonic \\
\hline & Catatonic & & Melancholic \\
\hline & Melancholic & & Atypical \\
\hline & Atypical & & Anxiety \\
\hline & Postpartum onset & & Suicide risk severity \\
\hline $.3 \mathrm{x}$ & Recurrent & & Postpartum onset \\
\hline \multirow[t]{3}{*}{$300.4 \mathrm{x}$} & Dysthymic disorder & D 02 & Major depressive disorder, recurrent \\
\hline & Specify if: early/late onset & D 03 & Chronic depressive disorder (dysthymia) \\
\hline & Specify: atypical features & D 04 & Premenstrual dysphoric disorder \\
\hline 311 & Depressive disorder NOS & D 05 & Mixed anxiety/depression \\
\hline 293.83 & Mood disorder due to a general medical condition & D 06 & Substance-induced depressive disorder \\
\hline \multirow[t]{7}{*}{ 29x.xx } & Substance-induced mood disorder & $\mathrm{D} 07$ & Depressive disorder associated with a known general medical condition \\
\hline & Appendix B (other mood disorders) & D 08 & Other specified depressive disorder \\
\hline & Premenstrual dysphoric disorder & D 09 & Unspecified depressive disorder \\
\hline & Alternative criterion B for dysthymic disorder & & \\
\hline & Minor depressive disorder & & \\
\hline & Recurrent brief depressive disorder & & \\
\hline & Mixed anxiety-depressive disorder & & \\
\hline \multicolumn{4}{|c|}{$\begin{array}{l}\text { "Based on the American Psychiatric Association. }{ }^{1,9} \\
\text { ban "x" appearing in a diagnostic code indicates that the specific code required for that diagnosis depends on the chronicity and severity of the } \\
\text { mood disorder. } \\
\text { Abbreviations: DSM = Diagnostic and Statistical Manual of Mental Disorders, NOS = not otherwise specified. }\end{array}$} \\
\hline
\end{tabular}

Figure 2. A Timeline of the Evolution of Antidepressant Treatments

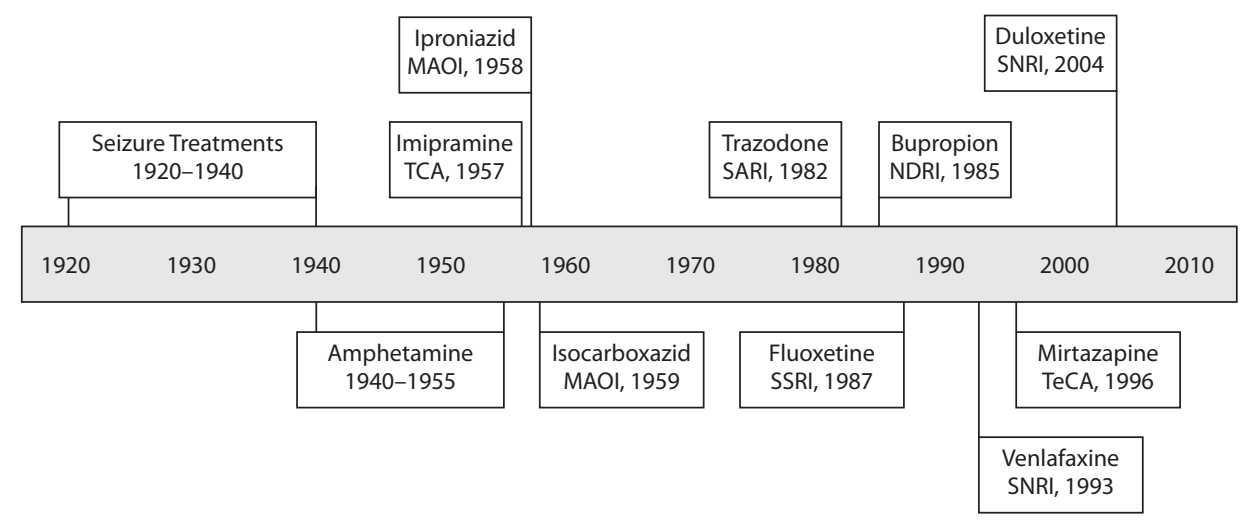

Abbreviations: $\mathrm{MAOI}=$ monoamine oxidase inhibitor, NDRI = norepinephrine-dopamine reuptake inhibitor, $\mathrm{SARI}=$ serotonin agonist and reuptake inhibitor, SNRI = serotonin-norepinephrine reuptake inhibitor, SSRI = selective serotonin reuptake inhibitor, TCA = tricyclic antidepressant, TeCA = tetracyclic antidepressant.

\section{Amphetamine}

In the 1930s, amphetamine became the first antidepressant medicine of the 20 th century. ${ }^{12}$ It was originally developed in 1929 as a decongestant for the treatment of asthma and hay fever in the United States. Amphetamine was found ineffective for these disorders but was observed to produce feelings of exhilaration and wakefulness. After being tested for a variety of disorders, including schizophrenia and anxiety, amphetamine was found to be effective in relieving depression, particularly with anhedonia. ${ }^{13}$ Positive clinical trials comparing amphetamine with placebo led to approval for the treatment of mood elevation in depression and other psychiatric conditions by the American Medical Association (AMA) Council on Pharmacy in 1937, with inclusion in the AMA prescribing guidelines. By the mid-1940s, amphetamine (marketed as Benzedrine) had become widely accepted beyond the inpatient arena, and an estimated 1 million or more amphetamine tablets were being consumed daily in the United States for not only mood elevation but also weight loss. ${ }^{12}$ However, nonprescription abuse of amphetamine was also widespread. Safety concerns as well as the introduction of new drugs in the 1960s led to the decline of amphetamine as a depression treatment. Although no longer used, amphetamine was the first drug treatment to be widely used for the long-term treatment of outpatients with depression both in psychiatry and in general practice and is therefore considered to be the first antidepressant. 


\section{Tricyclic Antidepressants}

During World War II, a search for antimalarial drugs led to the development of new agents, ushering in modern psychopharmacology. ${ }^{14,15}$ The phenothiazine dye methylene blue was effective for malaria but had undesirable side effects; it was modified to produce chlorpromazine in 1950. ${ }^{16,17}$ Although ineffective for malaria, chlorpromazine produced a calm and relaxed state. ${ }^{18}$ Jean Delay and Pierre Deniker in France began to treat patients with schizophrenia with chlorpromazine and found that it had antihistamine properties. ${ }^{17,18}$

In 1956, a compound closely related chemically to chlorpromazine, imipramine, was found to improve mood in patients with depression by Roland Kuhn in Switzerland, who published the results in $1957 .{ }^{18}$ The development of several other tricyclic antidepressants (TCAs) soon followed.

The putative antidepressant mechanism of action of TCAs is to block the reuptake of specific neurotransmittersparticularly serotonin and norepinephrine-in the synapse between neurons. ${ }^{18}$

The TCA class of medication came to be a first-line treatment for depression and was widely used for more than 30 years. ${ }^{15}$

\section{Monoamine Oxidase Inhibitors}

Also in the 1950s, investigations by several groups and individuals led to the development of monoamine oxidase inhibitors (MAOIs) as antidepressants. ${ }^{18}$ George Crane of the United States observed that iproniazid substantially improved mood in patients who were treated for tuberculosis. ${ }^{19}$ The MAOI properties were recognized because iproniazid was known to reverse the depressive effects of reserpine, an antihypertensive agent which had been shown to deplete the central monoamines serotonin and norepinephrine.

The mechanism of action of the MAOIs differs from that of the TCAs, although both classes increase the amount of circulating monoamines in the synapse between neurons. The MAOIs increase synaptic catecholamines by inhibiting the monoamine oxidase enzyme that metabolizes catecholamines.

Iproniazid caused substantial liver toxicity ${ }^{20}$ and was eventually discontinued, but similar drugs that were less toxic, such as tranylcypromine and phenelzine, were developed. ${ }^{21}$ Tranylcypromine was discovered in 1959 and approved for use in the United States in 1961 but was later withdrawn because of hypertensive crises and intracranial bleeding. When tranylcypromine was reintroduced, stricter treatment guidelines were outlined. ${ }^{22}$ In many ways, MAOIs may be the most efficacious medications available to treat depression, but their widespread use has been limited by drug interactions, dietary restrictions, and side effects. ${ }^{23}$

\section{The Catecholamine Hypothesis}

The presumed mechanism of action of the TCAs and MAOIs led scientists to develop a theory of the pathophysiology of depression in 1965: the catecholamine hypothesis. ${ }^{23,24}$ Simply put, depression results from a deficiency of catecholamines (particularly norepinephrine) in synapses in the brain, while elation results from an excess of these amines.

\section{Mixed-Action Antidepressants}

In the 1980s and 1990s, medications with mechanisms of action that act on more than one neurotransmitter were developed. ${ }^{25}$ Trazodone and nefazodone inhibit norepinephrine and serotonin reuptake, as well as act as antagonists of serotonin.

Trazodone was widely prescribed following its introduction to the market in 1982, but its use declined substantially after selective serotonin reuptake inhibitors (SSRIs) became available. Nefazodone has a similar chemical structure to trazodone; it antagonizes serotonin $5-\mathrm{HT}_{2}$ receptors and weakly inhibits serotonin and norepinephrine reuptake. ${ }^{26}$ Its tolerability profile is fairly benign and does not share the sexual side effects of the SSRIs. However, several case reports of nefazodone-induced liver failure led to its being withdrawn in the United States. ${ }^{26}$

Bupropion has a novel mechanism of action, different from that of TCAs and of MAOIs. Its mechanism of action is unclear, but it is thought to involve some inhibition of norepinephrine and dopamine reuptake, but not serotonin. ${ }^{27}$ Its tolerability and side effect profile differs from those of many SSRIs in that bupropion has less sedation, no sexual side effects, and no weight gain. Concern about bupropion inducing seizures led to its withdrawal from the market in the United States in the late 1980s. It was reintroduced in 1989 following demonstration that it did not increase seizure potential at therapeutic doses.

\section{Selective Serotonin Reuptake Inhibitors}

A major change in the treatment of depression occurred with the development of SSRIs. These medications are specific inhibitors of serotonin reuptake into afferent neurons. ${ }^{28}$ The US Food and Drug Administration (FDA) approved fluoxetine as the first SSRI in late 1987, and the development of several other SSRIs quickly followed. ${ }^{29}$

The SSRIs do not necessarily offer increased efficacy over the TCAs or the MAOIs; they do, however, have attractive properties. Unlike TCAs, SSRIs are not lethal in overdose and do not cause cardiac arrhythmia problems. ${ }^{30}$ In general, the SSRIs cause less sedation and weight gain and have fewer anticholinergic side effects than earlier antidepressants ${ }^{30}$ and do not require dietary restrictions. Dose titration is generally simpler. These improved safety and tolerability profiles of SSRIs led to their widespread use.

The use of SSRIs surpassed that of all other antidepressant agents by the mid-1990s. Among US patients treated for depression with antidepressants in psychiatric, primary care, or other practices from 1993 to 1994, more than half of each group received SSRIs. ${ }^{31}$

\section{Dual Reuptake Inhibitors}

In the 1990s, several dual reuptake inhibitors were developed to target only norepinephrine and serotonin 
reuptake. The intent was to provide greater efficacy than SSRIs and cause fewer side effects than other agents that affect both serotonin and norepinephrine (eg, MAOIs and TCAs) by avoiding additional action at other receptors. ${ }^{32}$ The best known serotonin-norepinephrine reuptake inhibitors are venlafaxine, which was approved by the FDA in 1994, and duloxetine, which was approved in 2004. Venlafaxine was synthesized early in the 1980s. Its mechanism of action was similar to that of the TCAs, but it was found to be much more tolerable and safe. Duloxetine was synthesized at about the same time as venlafaxine but was not approved by the FDA for treatment of major depression until 2004. This delay was the result of problems finding the therapeutic dose range. ${ }^{33,34}$

\section{CONCLUSION}

Although depression continues to be a burdensome and difficult-to-treat illness, clinicians have a wide variety of treatment options for patients who present with depression. Treatment has evolved dramatically over the last century, and since the TCAs and MAOIs were developed, no truly novel mechanisms of action have arisen in the antidepressant armamentarium. Although many treatments were discovered serendipitously, the search for improved safety and tolerability has driven the recent evolution of treatment in an effort to increase patients' adherence. Some of the current treatment options are considerably underused, and clinicians should continue to be alert for new and effective treatments.

Drug names: bupropion (Wellbutrin, Aplenzin, and others), duloxetine (Cymbalta), fluoxetine (Prozac and others), imipramine (Tofranil and others), isocarboxazid (Marplan), mirtazapine (Remeron and others), phenelzine (Nardil), reserpine (Serpalan and others), tranylcypromine (Parnate and others), trazodone (Oleptro and others), venlafaxine (Effexor and others).

Disclosure of off-label usage: The author has determined that, to the best of his knowledge, no investigational information about pharmaceutical agents that is outside US Food and Drug Administration-approved labeling has been presented in this article.

\section{REFERENCES}

1. American Psychiatric Association. Diagnostic and Statistical Manual of Mental Disorders, Fourth Edition. Washington, DC: American Psychiatric Association; 1994.

2. Kessler RC, Birnbaum H, Bromet E, et al. Age differences in major depression: results from the National Comorbidity Survey Replication (NCS-R). Psychol Med. 2010;40(2):225-237.

3. Keller MB, Lavori PW, Mueller TI, et al. Time to recovery, chronicity, and levels of psychopathology in major depression: a 5-year prospective follow-up of 431 subjects. Arch Gen Psychiatry. 1992;49(10):809-816.

4. Mueller TI, Leon AC, Keller MB, et al. Recurrence after recovery from major depressive disorder during 15 years of observational follow-up. Am J Psychiatry. 1999;156(7):1000-1006.

5. Fichter MM, Kohlboeck G, Quadflieg N. The Upper Bavarian longitudinal community study 1975-2004, pt 2: long-term course and outcome of depression: a controlled study. Eur Arch Psychiatry Clin Neurosci. 2008;258(8):476-488.

6. Judd LL, Akiskal HS, Maser JD, et al. A prospective 12-year study of subsyndromal and syndromal depressive symptoms in unipolar major depressive disorders. Arch Gen Psychiatry. 1998;55(8):694-700.

7. World Health Organization. The Global Burden of Disease: 2004 Update. Geneva, Switzerland: WHO Press; 2008. http://www.who.int/healthinfo/ global_burden_disease/GBD_report_2004update_full.pdf. Accessed February 20, 2012.

8. Fiedorowicz JG, Endicott J, Leon AC, et al. Subthreshold hypomanic symptoms in progression from unipolar major depression to bipolar disorder. Am J Psychiatry. 2011;168(1):40-48.

9. American Psychiatric Association. DSM-5 development: depressive disorders. http://www.dsm5.org/ProposedRevision/Pages/ DepressiveDisorders.aspx. Published 2012. Accessed April 17, 2012.

10. Fink M. Meduna and the origins of convulsive therapy. Am J Psychiatry. 1984;141(9):1034-1041.

11. Sabbatini RME. The history of shock therapy in psychiatry. Brain Mind Electron Mag Neurosci. 1997;4. http://www.cerebromente.org.br/n04/ historia/shock_i.htm. Accessed April 17, 2012.

12. Rasmussen N. Making the first anti-depressant: amphetamine in American medicine, 1929-1950. J Hist Med Allied Sci. 2006;61(3): 288-323.

13. Guttmann E, Sargant W. Observations on Benzedrine. BMJ. 1937; 1(3984):1013-1015.

14. Callahan CM, Berrios GE. Reinventing Depression: A History of the Treatment of Depression in Primary Care, 1940-2004. New York, NY: Oxford University Press; 2005.

15. Nelson JC. Tricyclic and tetracyclic drugs. In: Schatzberg AF, Nemeroff CB, eds. The American Psychiatric Publishing Textbook of Psychopharmacology. 4th ed. Arlington, VA: American Psychiatric Press, Inc; 2009:263-288.

16. Nasrallah HA, Tandon R. Classic antipsychotic medications. In: Schatzberg AF, Nemeroff CB, eds. The American Psychiatric Publishing Textbook of Psychopharmacology. 4th ed. Arlington, VA: American Psychiatric Publishing; 2009:533-554.

17. Frankenburg FR, Baldessarini RJ. Neurosyphilis, malaria, and the discovery of antipsychotic agents. Harv Rev Psychiatry. 2008;16(5): 299-307.

18. Ban TA. The role of serendipity in drug discovery. Dialogues Clin Neurosci. 2006;8(3):335-344.

19. Crane GE. The psychiatric side-effects of iproniazid. Am J Psychiatry. 1956;112(7):494-501

20. Rosenblum LE, Korn RJ, Zimmerman HJ. Hepatocellular jaundice as a complication of iproniazid therapy. Arch Intern Med. 1960;105(4): 583-593.

21. Krishnan KRR. Monoamine oxidase inhibitors. In: Schatzberg AF, Nemeroff CB, eds. The American Psychiatric Publishing Textbook of Psychopharmacology. 4th ed. Arlington, VA: American Psychiatric Publishing; 2009:389-402.

22. Atchley DW. Reevaluation of tranylcypromine sulfate. JAMA. 1964; 189(10):763-764.

23. Fiedorowicz JG, Swartz KL. The role of monoamine oxidase inhibitors in current psychiatric practice. J Psychiatr Pract. 2004;10(4):239-248.

24. Schildkraut JJ. The catecholamine hypothesis of affective disorders: a review of supporting evidence. Am J Psychiatry. 1965;122(5):509-522.

25. Feighner JP. Mechanism of action of antidepressant medications. J Clin Psychiatry. 1999;60(suppl 4):4-11, discussion 12-13.

26. Golden RN, Dawkins K, Nicholas L. Trazodone and nefazodone. In: Schatzberg AF, Nemeroff CB, eds. The American Psychiatric Publishing Textbook of Psychopharmacology. 4th ed. Arlington, VA: American Psychiatric Publishing; 2009:403-414.

27. Clayton AH, Gillespie EH. Bupropion. In: Schatzberg AF, Nemeroff CB, eds. The American Psychiatric Publishing Textbook of Psychopharmacology. 4th ed. Arlington, VA: American Psychiatric Publishing; 2009:415-428.

28. Zahajszky J, Rosenbaum JF, Tollefson GD. Fluoxetine. In: Schatzberg AF, Nemeroff CB, eds. The American Psychiatric Publishing Textbook of Psychopharmacology. 4th ed. Arlington, VA: American Psychiatric Publishing; 2009:289-306.

29. Owens MJ. Selectivity of antidepressants: from the monoamine hypothesis of depression to the SSRI revolution and beyond. J Clin Psychiatry. 2004;65(suppl 4):5-10.

30. Ferguson JM. SSRI antidepressant medications: adverse effects and tolerability. Prim Care Companion J Clin Psychiatry. 2001;3(1):22-27.

31. Pincus HA, Tanielian TL, Marcus SC, et al. Prescribing trends in psychotropic medications: primary care, psychiatry, and other medical specialties. JAMA. 1998;279(7):526-531.

32. Jain R. Single-action versus dual-action antidepressants. Prim Care Companion J Clin Psychiatry. 2004;6(suppl 1):7-11.

33. Thase ME, Sloan DM. Venlafaxine and desvenlafaxine. In: Schatzberg AF, Nemeroff CB, eds. The American Psychiatric Publishing Textbook of Psychopharmacology. 4th ed. Arlington, VA: American Psychiatric Publishing; 2009:439-452.

34. Norris S, Blier P. Duloxetine and milnacipran. In: Schatzberg AF, Nemeroff CB, eds. The American Psychiatric Publishing Textbook of Psychopharmacology. 4th ed. Arlington, VA: American Psychiatric Publishing; 2009:453-464. 